\title{
Behavior of solutions of a second order rational difference equation
}

\author{
RAAFAT ABO-ZEID
}

Abstract. In this paper, we solve the difference equation

$$
x_{n+1}=\frac{\alpha}{x_{n} x_{n-1}-1}, \quad n=0,1, \ldots,
$$

where $\alpha>0$ and the initial values $x_{-1}, x_{0}$ are real numbers. We find some invariant sets and discuss the global behavior of the solutions of that equation. We show that when $\alpha>\frac{2}{3 \sqrt{3}}$, under certain conditions there exist solutions, that are either periodic or converging to periodic solutions. We show also the existence of dense solutions in the real line. Finally, we show that when $\alpha<\frac{2}{3 \sqrt{3}}$, one of the negative equilibrium points attracts all orbits with initials outside a set of Lebesgue measure zero.

\section{INTRODUCTION}

In [9], Amleh et al. studied the difference equation

$$
x_{n+1}=\frac{\alpha}{x_{n} x_{n-1}+1}, \quad n=0,1, \ldots,
$$

where the $\alpha$ is positive and the initial conditions are nonnegative real numbers. They conjectured that every solution has a finite limit but confirmed it only when $\alpha \leq 2$.

In [8], The authors studied the difference equation

$$
x_{n+1}=\frac{\alpha}{1+x_{n} x_{n-1}+c x_{n-1}}, \quad n=0,1, \ldots,
$$

where the $\alpha$ is positive and the initial conditions are nonnegative real numbers. They conjectured that the unique positive equilibrium point is globally asymptotically stable and confirmed it only when $(\alpha-c)^{2} \leq 4$.

Kulenović et al. [24], studied equation (1) and gave a unified proof for all values of $\alpha$ that the unique equilibrium is globally asymptotically stable.

2010 Mathematics Subject Classification. Primary: 39A20; Secondary: 39A21.

Key words and phrases. Difference equation, forbidden set, periodic solution, unbounded solution.

Full paper. Received 11 April 2018, revised 17 December 2018, accepted 11 February 2019, available online 12 February 2019. 
For more on difference equations with quadratic terms, see [1]-[14], [16], [17], [19], [21]-[25], [27]-[29].

In this paper, we study the difference equation

$$
x_{n+1}=\frac{\alpha}{x_{n} x_{n-1}-1}, \quad n=0,1, \ldots,
$$

where $\alpha>0$ and the initial conditions are real numbers. The transformation

$$
x_{n}=\frac{y_{n-1}}{y_{n}}, \quad \text { with } \quad y_{-2}=1,
$$

reduces the difference equation (2) into the linear third order difference equation

$$
y_{n+1}+\frac{1}{\alpha} y_{n}-\frac{1}{\alpha} y_{n-2}=0, \quad n=0,1, \ldots
$$

The characteristic equation of equation (3) is

$$
\lambda^{3}+\frac{1}{\alpha} \lambda^{2}-\frac{1}{\alpha}=0
$$

Clear that equation (4) has a positive real root $\lambda_{0}$ for all values of $\alpha$.

Equation (4) can be written as

$$
\lambda^{3}+\frac{1}{\alpha} \lambda^{2}-\frac{1}{\alpha}=\left(\lambda-\lambda_{0}\right)\left(\lambda^{2}+\left(\lambda_{0}+\frac{1}{\alpha}\right) \lambda+\lambda_{0}\left(\lambda_{0}+\frac{1}{\alpha}\right)\right)=0 .
$$

Therefore, the roots of equation (4) are

$$
\lambda_{0}, \quad \lambda_{ \pm}=-\frac{\lambda_{0}+\frac{1}{\alpha}}{2} \pm \frac{\sqrt{\left(\lambda_{0}+\frac{1}{\alpha}\right)^{2}-4 \lambda_{0}\left(\lambda_{0}+\frac{1}{\alpha}\right)}}{2} .
$$

We have the following result:

Lemma 1.1. For equation (4), we have the following:

(1) If $\alpha>\frac{2}{3 \sqrt{3}}$, then equation (4) has one positive real root and two complex conjugate roots.

(2) If $\alpha=\frac{2}{3 \sqrt{3}}$, then equation (4) has one positive real root and a repeated negative real root.

(3) If $\alpha<\frac{2}{3 \sqrt{3}}$, then equation (4) has three real different roots, one of them is positive and two negative roots.

Proof. It is sufficient to see that, the discriminant of the polynomial

$$
p(\lambda)=\lambda^{3}+\frac{1}{\alpha} \lambda^{2}-\frac{1}{\alpha}=0
$$

is

$$
\triangle=-4 \frac{1}{\alpha^{4}}+27 \frac{1}{\alpha^{2}}
$$




\section{Forbidden SET AND SOlution OF EQUATIOn (2)}

As the solution of equation (2) depends on $\alpha$, we shall consider the three cases given in Lemma 1.1.

Case $\alpha>\frac{2}{3 \sqrt{3}}$ :

When $\alpha>\frac{2}{3 \sqrt{3}}$, the roots of equation (4) are

$$
\lambda_{0} \text { and } \lambda_{ \pm}=-\frac{\lambda_{0}+\frac{1}{\alpha}}{2} \pm i \frac{\sqrt{4 \lambda_{0}\left(\lambda_{0}+\frac{1}{\alpha}\right)-\left(\lambda_{0}+\frac{1}{\alpha}\right)^{2}}}{2} .
$$

Then the solution of equation (2) is

$$
x_{n}=\frac{c_{1} \lambda_{0}^{n-1}+\left(\frac{1}{\alpha \lambda_{0}}\right)^{\frac{n-1}{2}}\left(c_{2} \cos (n-1) \theta+c_{3} \sin (n-1) \theta\right)}{c_{1} \lambda_{0}^{n}+\left(\frac{1}{\alpha \lambda_{0}}\right)^{\frac{n}{2}}\left(c_{2} \cos n \theta+c_{3} \sin n \theta\right)},
$$

where

$$
\begin{aligned}
\left|\lambda_{ \pm}\right| & =\sqrt{\lambda_{0}\left(\lambda_{0}+\frac{1}{\alpha}\right)}=\sqrt{\frac{1}{\alpha \lambda_{0}}} \\
\theta & \left.=\tan ^{-1}\left(-\sqrt{\frac{3 \lambda_{0} \alpha-1}{\lambda_{0} \alpha+1}}\right) \in\right] \frac{\pi}{2}, \pi[.
\end{aligned}
$$

Using the initials $y_{-2}, y_{-1}$ and $y_{0}$, the values of $c_{1}, c_{2}$ and $c_{3}$ are:

$$
\begin{aligned}
& c_{1}=\left(y_{0} c_{11}+y_{-1} c_{12}+y_{-2} c_{13}\right), \\
& c_{2}=\left(y_{0} c_{21}+y_{-1} c_{22}+y_{-2} c_{23}\right), \\
& c_{3}=\left(y_{0} c_{31}+y_{-1} c_{32}+y_{-2} c_{33}\right),
\end{aligned}
$$

where

$$
\begin{aligned}
& c_{11}=-\frac{1}{\Delta_{1}} \lambda_{0} \alpha \sqrt{\lambda_{0} \alpha} \sin \theta, \\
& c_{12}=\frac{1}{\Delta_{1}} \lambda_{0} \alpha \sin 2 \theta, \\
& c_{13}=-\frac{1}{\Delta_{1}} \sqrt{\lambda_{0} \alpha} \sin \theta, \\
& c_{21}=\frac{1}{\Delta_{1}}\left(\alpha \sin 2 \theta-\frac{\sqrt{\lambda_{0} \alpha}}{\lambda_{0}^{2}} \sin \theta\right), \quad c_{22}=-\frac{1}{\Delta_{1}} \lambda_{0} \alpha \sin 2 \theta, \\
& c_{23}=-\frac{1}{\Delta_{1}} \sqrt{\lambda_{0} \alpha} \sin \theta \text {, } \\
& c_{31}=\frac{1}{\Delta_{1}}\left(\alpha \cos 2 \theta-\frac{\sqrt{\lambda_{0} \alpha}}{\lambda_{0}^{2}} \cos \theta\right) \\
& c_{32}=\frac{1}{\Delta_{1}}\left(-\lambda_{0} \alpha \cos 2 \theta+\frac{1}{\lambda_{0}^{2}}\right), \\
& c_{33}=\frac{1}{\Delta_{1}}\left(\sqrt{\lambda_{0} \alpha} \cos \theta-\frac{1}{\lambda_{0}}\right),
\end{aligned}
$$

and

$$
\Delta_{1}=\left|\begin{array}{ccc}
1 & 1 & 0 \\
\frac{1}{\lambda_{0}} & \sqrt{\lambda_{0} \alpha} \cos \theta & -\sqrt{\lambda_{0} \alpha} \sin \theta \\
\frac{1}{\lambda_{0}^{2}} & \lambda_{0} \alpha \cos 2 \theta & -\lambda_{0} \alpha \sin 2 \theta
\end{array}\right| .
$$

By simple calculations, we can write the solution of equation (3) as

$$
y_{n}=\gamma_{1 n} y_{0}+\gamma_{2 n} y_{-1}+\gamma_{3 n} y_{-2},
$$


where

$$
\begin{aligned}
& \gamma_{1 n}=c_{11} \lambda_{0}^{n}+c_{21}\left(\frac{1}{\lambda_{0} \alpha}\right)^{\frac{n}{2}} \cos n \theta+c_{31}\left(\frac{1}{\lambda_{0} \alpha}\right)^{\frac{n}{2}} \sin n \theta, \\
& \gamma_{2 n}=c_{12} \lambda_{0}^{n}+c_{22}\left(\frac{1}{\lambda_{0} \alpha}\right)^{\frac{n}{2}} \cos n \theta+c_{32}\left(\frac{1}{\lambda_{0} \alpha}\right)^{\frac{n}{2}} \sin n \theta \\
& \gamma_{3 n}=c_{13} \lambda_{0}^{n}+c_{23}\left(\frac{1}{\lambda_{0} \alpha}\right)^{\frac{n}{2}} \cos n \theta+c_{33}\left(\frac{1}{\lambda_{0} \alpha}\right)^{\frac{n}{2}} \sin n \theta
\end{aligned}
$$

are such that $c_{i j}, i, j=1,2,3$ are given in (7).

Case $\alpha=\frac{2}{3 \sqrt{3}}$ :

When $\alpha=\frac{2}{3 \sqrt{3}}$, the roots of equation (4) are

$$
\lambda_{0}=\frac{1}{3 \alpha}, \quad-\frac{2}{3 \alpha}, \quad-\frac{2}{3 \alpha} .
$$

Then the solution of equation (2) is

$$
x_{n}=\frac{c_{1}\left(\frac{1}{3 \alpha}\right)^{n-1}+c_{2}\left(-\frac{2}{3 \alpha}\right)^{n-1}+c_{3}\left(-\frac{2}{3 \alpha}\right)^{n-1}(n-1)}{c_{1}\left(\frac{1}{3 \alpha}\right)^{n}+c_{2}\left(-\frac{2}{3 \alpha}\right)^{n}+c_{3}\left(-\frac{2}{3 \alpha}\right)^{n} n} .
$$

Using the initials $y_{-2}, y_{-1}$ and $y_{0}$, the values of $c_{1}, c_{2}$ and $c_{3}$ in this case are:

$$
\begin{aligned}
& c_{1}=y_{0} c_{11}+y_{-1} c_{12}+y_{-2} c_{13}, \\
& c_{2}=y_{0} c_{21}+y_{-1} c_{22}+y_{-2} c_{23}, \\
& c_{3}=y_{0} c_{31}+y_{-1} c_{32}+y_{-2} c_{33},
\end{aligned}
$$

where

$$
\begin{aligned}
& c_{11}=\frac{1}{\Delta_{2}} \frac{27}{8} \alpha^{3}, \quad c_{12}=\frac{1}{\Delta_{2}} \frac{9}{2} \alpha^{2}, \quad c_{13}=\frac{1}{\Delta_{2}} \frac{3}{2} \alpha, \\
& c_{21}=\frac{1}{\Delta_{2}} 27 \alpha^{3}, \quad c_{22}=-\frac{9}{2} \alpha^{2}, \quad c_{23}=-\frac{1}{\Delta_{2}} \frac{3}{2} \alpha, \\
& c_{31}=\frac{1}{\Delta_{2}} \frac{81}{4} \alpha^{3}, \quad c_{32}=\frac{1}{\Delta_{2}} \frac{27}{4} \alpha^{2}, \quad c_{33}=-\frac{1}{\Delta_{2}} \frac{9}{2} \alpha
\end{aligned}
$$

and

$$
\Delta_{2}=\left|\begin{array}{ccc}
1 & 1 & 0 \\
3 \alpha & -\frac{3}{2} \alpha & \frac{3}{2} \alpha \\
(3 \alpha)^{2} & \left(-\frac{3 \alpha}{2}\right)^{2} & -2\left(-\frac{3 \alpha}{2}\right)^{2}
\end{array}\right|
$$

By simple calculations, we can write the solution of equation (3) in this case as

$$
y_{n}=\gamma_{1 n} y_{0}+\gamma_{2 n} y_{-1}+\gamma_{3 n} y_{-2},
$$

where 


$$
\begin{aligned}
& \gamma_{1 n}=c_{11}\left(\frac{1}{3 \alpha}\right)^{n}+c_{21}\left(-\frac{2}{3 \alpha}\right)^{n}+c_{31}\left(-\frac{2}{3 \alpha}\right)^{n} n, \\
& \gamma_{2 n}=c_{12}\left(\frac{1}{3 \alpha}\right)^{n}+c_{22}\left(-\frac{2}{3 \alpha}\right)^{n}+c_{32}\left(-\frac{2}{3 \alpha}\right)^{n} n, \\
& \gamma_{3 n}=c_{13}\left(\frac{1}{3 \alpha}\right)^{n}+c_{23}\left(-\frac{2}{3 \alpha}\right)^{n}+c_{33}\left(-\frac{2}{3 \alpha}\right)^{n} n
\end{aligned}
$$

are such that $c_{i j}, i, j=1,2,3$ are given in (9).

Case $\alpha<\frac{2}{3 \sqrt{3}}$ :

When $\alpha<\frac{2}{3 \sqrt{3}}$, the roots of equation (4) are

$$
\lambda_{0} \text { and } \lambda_{ \pm}=-\frac{\lambda_{0}+\frac{1}{\alpha}}{2} \pm \frac{\sqrt{\left(\lambda_{0}+\frac{1}{\alpha}\right)^{2}-4 \lambda_{0}\left(\lambda_{0}+\frac{1}{\alpha}\right)}}{2},
$$

where

$$
0<\lambda_{0}<\left|\lambda_{+}\right|<\left|\lambda_{-}\right| .
$$

Then the solution of equation (2) is

$$
x_{n}=\frac{c_{1} \lambda_{0}^{n-1}+c_{2} \lambda_{-}^{n-1}+c_{3} \lambda_{+}^{n-1}}{c_{1} \lambda_{0}^{n}+c_{2} \lambda_{-}^{n}+c_{3} \lambda_{+}^{n}} .
$$

Using the initials $y_{-2}, y_{-1}$ and $y_{0}$, the values of $c_{1}, c_{2}$ and $c_{3}$ in this case are:

$$
\begin{aligned}
& c_{1}=y_{0} c_{11}+y_{-1} c_{12}+y_{-2} c_{13}, \\
& c_{2}=y_{0} c_{21}+y_{-1} c_{22}+y_{-2} c_{23}, \\
& c_{3}=y_{0} c_{31}+y_{-1} c_{32}+y_{-2} c_{33},
\end{aligned}
$$

where

$$
\begin{aligned}
& c_{11}=\frac{1}{\Delta_{3}} \frac{\lambda_{-}-\lambda_{+}}{\lambda_{-}^{2} \lambda_{+}^{2}}, \quad c_{12}=\frac{1}{\Delta_{3}} \frac{-\lambda_{-}^{2}+\lambda_{+}^{2}}{\lambda_{-}^{2} \lambda_{+}^{2}}, \quad c_{13}=\frac{1}{\Delta_{3}} \frac{\lambda_{-}-\lambda_{+}}{\lambda_{-} \lambda_{+}}, \\
& c_{21}=\frac{1}{\Delta_{3}} \frac{\lambda_{+}-\lambda_{0}}{\lambda_{+}^{2} \lambda_{0}^{2}}, \quad c_{22}=\frac{1}{\Delta_{3}} \frac{\lambda_{0}^{2}-\lambda_{+}^{2}}{\lambda_{+}^{2} \lambda_{0}^{2}}, \quad c_{23}=\frac{1}{\Delta_{3}} \frac{\lambda_{+}-\lambda_{0}}{\lambda_{+} \lambda_{0}}, \\
& c_{31}=\frac{1}{\Delta_{3}} \frac{\lambda_{0}-\lambda_{-}}{\lambda_{0}^{2} \lambda_{-}^{2}}, \quad c_{32}=\frac{1}{\Delta_{3}} \frac{\lambda_{-}^{2}-\lambda_{0}^{2}}{\lambda_{0}^{2} \lambda_{-}^{2}}, \quad c_{33}=\frac{1}{\Delta_{3}} \frac{\lambda_{0}-\lambda_{-}}{\lambda_{0} \lambda_{-}}
\end{aligned}
$$

and

$$
\Delta_{3}=\left|\begin{array}{ccc}
1 & 1 & 1 \\
\frac{1}{\lambda_{0}} & \frac{1}{\lambda_{-}} & \frac{1}{\lambda_{+}} \\
\frac{1}{\lambda_{0}^{2}} & \frac{1}{\lambda_{-}^{2}} & \frac{1}{\lambda_{+}^{2}}
\end{array}\right| .
$$

By simple calculations, we can write the solution of equation (3) in this case as

$$
y_{n}=\gamma_{1 n} y_{0}+\gamma_{2 n} y_{-1}+\gamma_{3 n} y_{-2},
$$

where 


$$
\begin{aligned}
\gamma_{1 n} & =c_{11} \lambda_{0}^{n}+c_{21} \lambda_{-}^{n}+c_{31} \lambda_{+}^{n}, \\
\gamma_{2 n} & =c_{12} \lambda_{0}^{n}+c_{22} \lambda_{-}^{n}+c_{32} \lambda_{+}^{n} \\
\gamma_{3 n} & =c_{13} \lambda_{0}^{n}+c_{23} \lambda_{-}^{n}+c_{33} \lambda_{+}^{n}
\end{aligned}
$$

are such that $c_{i j}, i, j=1,2,3$ are given in (11).

Using the previous arguments we can give the form of the forbidden set in the following result.

Theorem 2.1. The forbidden set of equation (2) as

$$
F=\bigcup_{n=-1}^{\infty}\left\{\left(x_{0}, x_{-1}\right) \in \mathbb{R}^{2}: \frac{\gamma_{1 n}}{x_{0} x_{-1}}+\frac{\gamma_{2 n}}{x_{-1}}+\gamma_{3 n}=0\right\},
$$

where $\gamma_{1 n}, \gamma_{2 n}$ and $\gamma_{3 n}$ are given as follows:

$$
\begin{cases}\gamma_{1 n}, \gamma_{2 n} \text { and } \gamma_{3 n} \text { are given in }(8), & \alpha>\frac{2}{3 \sqrt{3}} \\ \gamma_{1 n}, \gamma_{2 n} \text { and } \gamma_{3 n} \text { are given in }(10), & \alpha=\frac{2}{3 \sqrt{3}} \\ \gamma_{1 n}, \gamma_{2 n} \text { and } \gamma_{3 n} \text { are given in }(12), & \alpha<\frac{2}{3 \sqrt{3}} .\end{cases}
$$

Proof. The transformation

$$
x_{n}=\frac{y_{n-1}}{y_{n}}, \quad \text { with } \quad y_{-2}=1,
$$

reduces the difference equation (2) into the linear third order difference equation

$$
y_{n+1}+\frac{1}{\alpha} y_{n}-\frac{1}{\alpha} y_{n-2}=0, \quad n=0,1, \ldots
$$

This equation has the characteristic equation

$$
\lambda^{3}+\frac{1}{\alpha} \lambda^{2}-\frac{1}{\alpha}=0
$$

The solution of the characteristic equation depends on the value of its discriminant according to Lemma (1.1).

When $\alpha>\frac{2}{3 \sqrt{3}}$, the roots of the characteristic equation are

$$
\lambda_{0} \text { and } \lambda_{ \pm}=-\frac{\lambda_{0}+\frac{1}{\alpha}}{2} \pm i \frac{\sqrt{4 \lambda_{0}\left(\lambda_{0}+\frac{1}{\alpha}\right)-\left(\lambda_{0}+\frac{1}{\alpha}\right)^{2}}}{2}
$$

where $\lambda_{0}$ is a positive real root.

Then

where

$$
y_{n}=c_{1} \lambda_{0}^{n}+\left(\frac{1}{\alpha \lambda_{0}}\right)^{\frac{n}{2}}\left(c_{2} \cos n \theta+c_{3} \sin n \theta\right),
$$

$\left|\lambda_{ \pm}\right|=\sqrt{\lambda_{0}\left(\lambda_{0}+\frac{1}{\alpha}\right)}=\sqrt{\frac{1}{\alpha \lambda_{0}}} \quad$ and $\left.\quad \theta=\tan ^{-1}\left(-\sqrt{\frac{3 \lambda_{0} \alpha-1}{\lambda_{0} \alpha+1}}\right) \in\right] \frac{\pi}{2}, \pi[$. 
Using the initials $y_{-2}, y_{-1}$ and $y_{0}$, we can find the values of $c_{1}, c_{2}$ and $c_{3}$. These values are given in formulas (6). By simple calculations, we can write the solution

$$
y_{n}=\gamma_{1 n} y_{0}+\gamma_{2 n} y_{-1}+\gamma_{3 n} y_{-2},
$$

where $\gamma_{1 n}, \gamma_{2 n}$ and $\gamma_{3 n}$ are given in formulas (8).

But as

$$
y_{-1}=\frac{1}{x_{-1}}, y_{0}=\frac{1}{x_{0} x_{-1}},
$$

we can write

$$
y_{n}=\frac{\gamma_{1 n}}{x_{0} x_{-1}}+\frac{\gamma_{2 n}}{x_{-1}}+\gamma_{3 n} .
$$

Using the transformation

$$
x_{n}=\frac{y_{n-1}}{y_{n}}, \quad \text { with } \quad y_{-2}=1,
$$

it is clear that $x_{n}$ is well-defined whenever $y_{n} \neq 0, n \geq-1$.

Therefore, the forbidden set in this case is

$$
F=\bigcup_{n=-1}^{\infty}\left\{\left(x_{0}, x_{-1}\right) \in \mathbb{R}^{2}: \frac{\gamma_{1 n}}{x_{0} x_{-1}}+\frac{\gamma_{2 n}}{x_{-1}}+\gamma_{3 n}=0\right\},
$$

where $\gamma_{1 n}, \gamma_{2 n}$ and $\gamma_{3 n}$ are given in formulas (8).

When $\alpha=\frac{2}{3 \sqrt{3}}$ and $\alpha<\frac{2}{3 \sqrt{3}}$, the proof is similar and will be omitted. The proof is complete.

\section{INVARIANT SETS FOR EQUATION (2)}

In this section, we shall give invariant sets for equation (2).

When $\alpha>\frac{2}{3 \sqrt{3}}$, we can write the constant

$$
c_{1}=y_{0} c_{11}+y_{-1} c_{12}+y_{-2} c_{13}
$$

in terms of $x_{0}$ and $x_{-1}$ as

$$
c_{1}\left(x_{0}, x_{-1}\right)=\frac{1}{x_{0} x_{-1}} c_{11}+\frac{1}{x_{-1}} c_{12}+c_{13} .
$$

By simple calculations, we can show that if $\left(x_{0}, x_{-1}\right)$ is such that $c_{1}\left(x_{0}, x_{-1}\right)=$ 0 , then $\left(x_{0}, x_{-1}\right)$ lies on the rectangular hyperbola

$$
\frac{\alpha \lambda_{0}}{x_{0} x_{-1}}+\frac{1}{\lambda_{0} x_{-1}}+1=0 .
$$

Consider the set

$$
D_{1}=\left\{(x, y) \in \mathbb{R}^{2}: \frac{\alpha \lambda_{0}}{x y}+\frac{1}{\lambda_{0} y}+1=0\right\} .
$$

Theorem 3.1. The set $D_{1}$ is an invariant for equation (2). 
Proof. Let $\left(x_{0}, x_{-1}\right) \in D_{1}$. We show that $\left(x_{n}, x_{n-1}\right) \in D_{1}$ for each $n \in N$. The proof is by induction on $n$. The point $\left(x_{0}, x_{-1}\right) \in D_{1}$, implies

$$
\frac{\alpha \lambda_{0}}{x_{0} x_{-1}}+\frac{1}{\lambda_{0} x_{-1}}+1=0 .
$$

Now for $n=1$, we have

$$
\begin{aligned}
\frac{\alpha \lambda_{0}}{x_{1} x_{0}}+\frac{1}{\lambda_{0} x_{0}}+1 & =\frac{\alpha \lambda_{0}}{x_{0} \alpha}\left(x_{0} x_{-1}-1\right)+\frac{1}{\lambda_{0} x_{0}}+1 \\
& =\lambda_{0} x_{-1}+\frac{\lambda_{0}}{x_{0}}\left(-1+\frac{1}{\lambda_{0}^{2}}\right)+1 .
\end{aligned}
$$

But as $\lambda_{0}$ is a solution of equation (4), we have

$$
-1+\frac{1}{\lambda_{0}^{2}}=\alpha \lambda_{0} .
$$

Then

$$
\begin{aligned}
\frac{\alpha \lambda_{0}}{x_{1} x_{0}}+\frac{1}{\lambda_{0} x_{0}}+1 & =\lambda_{0} x_{-1}+\frac{\lambda_{0}^{2} \alpha}{x_{0}}+1 \\
& =\lambda_{0} x_{-1}\left(1+\frac{\lambda_{0} \alpha}{x_{0} x_{-1}}\right)+1 \\
& =\lambda_{0} x_{-1}\left(-\frac{1}{\lambda_{0} x_{-1}}\right)+1=0 .
\end{aligned}
$$

This implies that $\left(x_{1}, x_{0}\right) \in D_{1}$.

Suppose now that $\left(x_{n}, x_{n-1}\right) \in D_{1}$. That is

$$
\frac{\alpha \lambda_{0}}{x_{n} x_{n-1}}+\frac{1}{\lambda_{0} x_{n-1}}+1=0 \text {. }
$$

Then

$$
\begin{aligned}
\frac{\alpha \lambda_{0}}{x_{n+1} x_{n}}+\frac{1}{\lambda_{0} x_{n}}+1 & =\frac{\alpha \lambda_{0}}{x_{n} \alpha}\left(x_{n} x_{n-1}-1\right)+\frac{1}{\lambda_{0} x_{n}}+1 \\
& =\lambda_{0} x_{n-1}+\frac{\lambda_{0}}{x_{n}}\left(-1+\frac{1}{\lambda_{0}^{2}}\right)+1 \\
& =\lambda_{0} x_{n-1}+\frac{\lambda_{0}^{2} \alpha}{x_{n}}+1 \\
& =\lambda_{0} x_{n-1}\left(1+\frac{\lambda_{0} \alpha}{x_{n} x_{n-1}}\right)+1 \\
& =\lambda_{0} x_{n-1}\left(-\frac{1}{\lambda_{0} x_{n-1}}\right)+1=0 .
\end{aligned}
$$

Therefore, $\left(x_{n+1}, x_{n}\right) \in D_{1}$ and the proof is complete. 
Definition 3.1 ([30]). A subset $N \subset \mathbb{R}^{n}$ has lesbegue measure zero if for every $\epsilon>0$, there exists a collection $\left\{E_{1}, E_{2}, \ldots\right\}$ of Borel sets with

$$
N \subset \cup_{i=1}^{\infty} E_{i}, \quad \sum_{i=1}^{\infty} \mu\left(E_{i}\right)<\epsilon .
$$

For more on lesbegue measure, one can see [15, 18, 20, 26, 31].

Theorem 3.2. The set $D_{1}$ is of Lebesgue measure zero.

Proof. It is required to prove that the set

$$
\left\{(x, g(x)) \in \mathbb{R}^{2}: g(x)=-\frac{1}{\lambda_{0}}-\frac{\alpha \lambda_{0}}{x}\right\}
$$

is of Lebesgue measure zero. We can write

$$
D_{1}=S_{+} \cup S_{-},
$$

where

$$
S_{+}=\left\{(x, g(x)) \in \mathbb{R}^{2}, x>0\right\}
$$

and

$$
S_{-}=\left\{(x, g(x)) \in \mathbb{R}^{2}, x<0\right\} .
$$

We show that $S_{+}$is of Lebesgue measure zero. For, let

$$
S_{+}=S_{1} \cup S_{2},
$$

where

$$
S_{1}=\left\{(x, g(x)) \in \mathbb{R}^{2}, 1 \leq x<\infty\right\}
$$

and

$$
S_{2}=\left\{(x, g(x)) \in \mathbb{R}^{2}, 0<x \leq 1\right\} .
$$

Now consider the part $S_{1}^{n}$ of the set $S_{1}$ in the interval $[n, n+1]$. For $\epsilon>0$, there exists $\delta>0$ such that $|g(x)-g(y)|<\frac{\epsilon}{2^{n+2}}$ whenever $|x-y|<\delta$ for all $x, y \in[n, n+1]$.

Divide the interval $[n, n+1]$ into $k$ subinterval $\left[n+\frac{i-1}{k}, n+\frac{i}{k}\right], 1 \leq i \leq k$ with $\frac{1}{k}<\delta$. Then

$$
S_{1}^{n} \subset \cup_{i=1}^{k}\left[n+\frac{i-1}{k}, n+\frac{i}{k}\right] \times\left[g\left(n+\frac{i-1}{k}\right)-\frac{\epsilon}{2^{n+2}}, g\left(n+\frac{i-1}{k}\right)+\frac{\epsilon}{2^{n+2}}\right] .
$$

That is

$$
\begin{aligned}
\mu\left(S_{1}^{n}\right) & <\sum_{i=1}^{k} \mu\left(\left[n+\frac{i-1}{k}, n+\frac{i}{k}\right] \times\left[g\left(n+\frac{i-1}{k}\right)-\frac{\epsilon}{2^{n+2}}, g\left(n+\frac{i-1}{k}\right)+\frac{\epsilon}{2^{n+2}}\right]\right) \\
& =\sum_{i=1}^{k} \frac{1}{k} \frac{\epsilon}{2^{n+1}}=\frac{\epsilon}{2^{n+1}} .
\end{aligned}
$$

But

$$
S_{1}=\cup_{n=1}^{\infty} S_{1}^{n}
$$


Then

$$
\mu\left(S_{1}\right)=\sum_{n=1}^{\infty} \mu\left(S_{1}^{n}\right)<\sum_{n=1}^{\infty} \frac{\epsilon}{2^{n+1}}=\frac{\epsilon}{2} .
$$

Similarly we can show that $\mu\left(S_{2}\right)<\frac{\epsilon}{2}$. Therefore, $\mu\left(S_{+}\right)<\epsilon$ and $S_{+}$is of Lebesgue measure zero.

The proof that $S_{-}$is of Lebesgue measure zero is similar and will be omitted. Thus $D_{1}=S_{+} \cup S_{-}$is a union of two Lebesgue measure zero subsets is also of Lebesgue measure zero.

This completes the proof.

In case $\alpha=\frac{2}{3 \sqrt{3}}$, consider the following subsets:

$$
\begin{aligned}
& D_{1}=\left\{(x, y) \in \mathbb{R}^{2}: \frac{1}{3 x y}+\frac{2}{\sqrt{3} y}+1=0\right\}, \\
& D_{2}=\left\{(x, y) \in \mathbb{R}^{2}: \frac{8}{3 x y}-\frac{2}{\sqrt{3} y}-1=0\right\}, \\
& D_{3}=\left\{(x, y) \in \mathbb{R}^{2}: \frac{2}{3 x y}+\frac{1}{\sqrt{3} y}-1=0\right\} .
\end{aligned}
$$

Note that $D_{1}, D_{2}$ and $D_{3}$ are equivalent to $c_{1}(x, y)=0, c_{2}(x, y)=0$ and $c_{3}(x, y)=0$ respectively. The two subsets $D_{1}$ and $D_{3}$ are invariant subsets for equation (2).

Finally when $\alpha<\frac{2}{3 \sqrt{3}}$. We shall consider the three sets

$$
D_{i}=\left\{(x, y) \in \mathbb{R}^{2}: \frac{\alpha \lambda}{x y}+\frac{1}{\lambda y}+1=0\right\}, \quad i=1,2,3,
$$

where

$$
\begin{cases}\lambda=\lambda_{0}, & i=1 \\ \lambda=\lambda_{-}, & i=2 \\ \lambda=\lambda_{+}, & i=3 .\end{cases}
$$

By simple calculations, we can see that:

$$
\left\{\begin{array}{lll}
D_{i} \text { is equivalent to } & c_{1}(x, y)=0, & i=1 \\
D_{i} \text { is equivalent to } & c_{2}(x, y)=0, & i=2 \\
D_{i} \text { is equivalent to } & c_{1}(x, y)=0, & i=3 .
\end{array}\right.
$$

Theorem 3.3. Each set of the sets $D_{i}, i=1,2$ and 3 is an invariant for equation (2).

Proof. The proof is similar to that of theorem (3.1) and will be omitted. 


\section{Global Behavior of equation (2)}

In this section, we shall investigate the behavior of equation (2) for all values of $\alpha$.

In the following results, we show that when $\alpha>\frac{2}{3 \sqrt{3}}$, under certain conditions there exist solutions, that are either periodic or converging to periodic solutions for equation (2).

Suppose that $\theta=\frac{p}{q} \pi$ is a rational multiple of $\pi$, where $p$ and $q$ are positive relatively prime integers such that $\frac{q}{2}<p<q$.

Theorem 4.1. Assume that $\alpha>\frac{2}{3 \sqrt{3}}$ and let $\left(x_{0}, x_{-1}\right) \notin F$. Then we have the following:

(1) If $\left(x_{0}, x_{-1}\right) \notin D_{1}$, then $\left\{x_{n}\right\}_{n=-1}^{\infty}$ converges to a periodic solution with prime period $q$.

(2) If $\left(x_{0}, x_{-1}\right) \in D_{1}$, then $\left\{x_{n}\right\}_{n=-1}^{\infty}$ is a periodic solution with prime period $q$.

Proof. When $\alpha>\frac{2}{3 \sqrt{3}}$, the solution of equation (2) can be written

$$
x_{q m+i}=\frac{c_{1} \lambda_{0}^{q m+i-1}+\left(\frac{1}{\alpha \lambda_{0}}\right)^{\frac{q m+i-1}{2}}\left(c_{2} \cos (q m+i-1) \theta+c_{3} \sin (q m+i-1) \theta\right)}{c_{1} \lambda_{0}^{q m+i}+\left(\frac{1}{\alpha \lambda_{0}}\right)^{\frac{q m+i}{2}}\left(c_{2} \cos (q m+i) \theta+c_{3} \sin (q m+i) \theta\right)},
$$

with $1 \leq i \leq q$.

(1) For each $i=1,2, \ldots, q$, we get

$$
x_{q m+i}=\sqrt{\lambda_{0} \alpha} \frac{c_{1}\left(\lambda_{0}^{3} \alpha\right)^{\frac{q m+i-1}{2}}+c_{2} \cos (p m \pi+(i-1) \theta)+c_{3} \sin (p m \pi+(i-1) \theta)}{c_{1}\left(\lambda_{0}^{3} \alpha\right)^{\frac{q m+i}{2}}+c_{2} \cos (p m \pi+i \theta)+c_{3} \sin (p m \pi+i \theta)} .
$$

As $m \rightarrow \infty$, we get

$$
x_{q m+i} \rightarrow \sqrt{\lambda_{0} \alpha} \frac{c_{2} \cos (i-1) \theta+c_{3} \sin (i-1) \theta}{c_{2} \cos i \theta+c_{3} \sin i \theta} .
$$

(2) Assume that $\left(x_{0}, x_{-1}\right) \in D_{1}$. Then for each $i=1,2, \ldots, q$, we get

$$
\begin{aligned}
x_{q m+i} & =\sqrt{\lambda_{0} \alpha} \frac{c_{2} \cos (q m+i-1) \theta+c_{3} \sin (q m+i-1) \theta}{c_{2} \cos (q m+i) \theta+c_{3} \sin (q m+i) \theta} \\
& =\sqrt{\lambda_{0} \alpha} \frac{c_{2} \cos (p m \pi+(i-1) \theta)+c_{3} \sin (p m \pi+(i-1) \theta)}{c_{2} \cos (p m \pi+i \theta)+c_{3} \sin (p m \pi+i \theta)} \\
& =\sqrt{\lambda_{0} \alpha} \frac{c_{2} \cos (i-1) \theta+c_{3} \sin (i-1) \theta}{c_{2} \cos i \theta+c_{3} \sin i \theta} .
\end{aligned}
$$

Suppose that $\theta \in \mathbb{R}-\pi \mathbb{Q}$ is not a rational multiple of $\pi$.

Theorem 4.2. Assume that $\alpha>\frac{2}{3 \sqrt{3}}$. If $\theta \in \mathbb{R}-\pi \mathbb{Q}$ is not a rational multiple of $\pi$, then the solution $\left\{x_{n}\right\}_{n=-1}^{\infty}$ is dense in $\mathbb{R}$. 
Proof. We can write the solution (5) of equation (2) as

$$
x_{n}=\sqrt{\lambda_{0} \alpha} \frac{c_{1}\left(\lambda_{0}^{3} \alpha\right)^{\frac{n-1}{2}}+A \sin ((n-1) \theta+\varphi)}{c_{1}\left(\lambda_{0}^{3} \alpha\right)^{\frac{n}{2}}+A \sin (n \theta+\varphi)},
$$

where $A=\sqrt{c_{2}^{2}+c_{3}^{2}}$ and $\left.\varphi=\tan ^{-1} \frac{c_{2}}{c_{3}} \in\right]-\frac{\pi}{2}, \frac{\pi}{2}\left[\right.$. Since $\frac{\theta}{\pi}$ is an irrational number, we can find for each $l \in \mathbb{R}$ a sequence $w_{k}=n_{k} \theta+\varphi-2 \pi m_{k}$, where $\left\{n_{k}\right\}_{k=1}^{\infty}$ and $\left\{m_{k}\right\}_{k=1}^{\infty}$ are sequences of positive integers such that

$$
\lim _{k \rightarrow \infty} w_{k}=l .
$$

Then

$$
x_{n_{k}}=\sqrt{\lambda_{0} \alpha} \frac{c_{1}\left(\lambda_{0}^{3} \alpha\right)^{\frac{n_{k}-1}{2}}+A \sin \left(w_{k}-\theta\right)}{c_{1}\left(\lambda_{0}^{3} \alpha\right)^{\frac{n_{k}}{2}}+A \sin w_{k}} .
$$

As $k \rightarrow \infty$, we get

$$
x_{n_{k}} \rightarrow \sqrt{\lambda_{0} \alpha} \frac{\sin (l-\theta)}{\sin l} .
$$

Now, consider the function

$$
\begin{aligned}
& f: \mathbb{R} / \pi \mathbb{Z} \rightarrow \mathbb{R}, \\
& x \mapsto \sqrt{\lambda_{0} \alpha} \frac{\sin (t-\theta)}{\sin t}, \text { where } t \notin \pi \mathbb{Z} .
\end{aligned}
$$

As the function is surjective, each number $z \in \mathbb{R}$ can be written as $\sqrt{\lambda_{0} \alpha} \frac{\sin (t-\theta)}{\sin t}$ for some $t \in \mathbb{R}$. Then each number $\sqrt{\lambda_{0} \alpha} \frac{\sin (t-\theta)}{\sin t} \in \mathbb{R}$ is a limit point of a sequence of the set $\left\{x_{n}: n \geq-1\right\}$.

This completes the proof.

Now returning to equation (2), where the equilibrium points depends on the values of $\gamma$. The equilibrium points are classified as the following:

- If $\alpha>\frac{2}{3 \sqrt{3}}$, then there is a unique positive equilibrium point $\bar{r}_{1}>\frac{2}{\sqrt{3}}$.

- If $\alpha=\frac{2}{3 \sqrt{3}}$, then there are two equilibrium points, $\bar{r}_{1}=\frac{2}{\sqrt{3}}$ and a negative equilibrium point $\bar{r}_{2}=-\frac{1}{\sqrt{3}}$.

- If $\alpha<\frac{2}{3 \sqrt{3}}$, then there are three equilibrium points, $\bar{r}_{1}<\frac{2}{\sqrt{3}}$ and two negative equilibrium points $\bar{r}_{2}$ and $\bar{r}_{3}$ such that

$$
\begin{aligned}
& \bar{r}_{2}=-\frac{\bar{r}_{1}}{2}+\frac{\sqrt{-3 \bar{r}_{1}^{2}+4}}{2}, \\
& \bar{r}_{3}=-\frac{\bar{r}_{1}}{2}-\frac{\sqrt{-3 \bar{r}_{1}^{2}+4}}{2}
\end{aligned}
$$

and

$$
\bar{r}_{3}<-\frac{1}{\sqrt{3}}<\bar{r}_{2}<0 .
$$


Theorem 4.3. Assume that $\alpha=\frac{2}{3 \sqrt{3}}$ and let $\left(x_{0}, x_{-1}\right) \notin F$. If $\left(x_{0}, x_{-1}\right) \notin$ $\cup_{i=1}^{3} D_{i}$, then the solution $\left\{x_{n}\right\}_{n=-1}^{\infty}$ converges to the negative equilibrium point $\bar{r}_{2}=-\frac{1}{\sqrt{3}}$.

Proof. We have that

$$
\begin{aligned}
x_{n} & =\frac{c_{1}\left(\frac{1}{3 \alpha}\right)^{n-1}+c_{2}\left(-\frac{2}{3 \alpha}\right)^{n-1}+c_{3}\left(-\frac{2}{3 \alpha}\right)^{n-1}(n-1)}{c_{1}\left(\frac{1}{3 \alpha}\right)^{n}+c_{2}\left(-\frac{2}{3 \alpha}\right)^{n}+c_{3}\left(-\frac{2}{3 \alpha}\right)^{n} n} \\
& =\frac{c_{1}\left(\frac{\sqrt{3}}{2}\right)^{n-1}+c_{2}(-\sqrt{3})^{n-1}+c_{3}(-\sqrt{3})^{n-1}(n-1)}{c_{1}\left(\frac{\sqrt{3}}{2}\right)^{n}+c_{2}(-\sqrt{3})^{n}+c_{3}(-\sqrt{3})^{n} n} \\
& =\frac{1}{\sqrt{3}} \frac{c_{1}\left(\frac{1}{2}\right)^{n-1}+c_{2}(-1)^{n-1}+c_{3}(-1)^{n-1}(n-1)}{c_{1}\left(\frac{1}{2}\right)^{n}+c_{2}(-1)^{n}+c_{3}(-1)^{n} n} .
\end{aligned}
$$

Clear that $x_{2 n}$ and $x_{2 n+1}$ converge to $-\frac{1}{\sqrt{3}}$, from which the result follows.

Note that: When $\alpha=\frac{2}{3 \sqrt{3}}$, the invariant subsets $D_{1}$ and $D_{3}$ intersect at $\left(-\frac{1}{\sqrt{3}},-\frac{1}{\sqrt{3}}\right)$.

Theorem 4.4. Assume that $\alpha<\frac{2}{3 \sqrt{3}}$. Then the equilibrium point $\overline{r_{2}}$ attracts all orbits with initial points outside a set of Lebesgue measure zero.

Proof. Suppose that $\left(x_{0}, x_{-1}\right) \notin F \cup\left(\cup_{i=1}^{3} D_{i}\right)$. The set of Lebesgue measure zero is the set $F \cup\left(\cup_{i=1}^{3} D_{i}\right)$. In fact it is a union of Lebesgue measure zero subsets of $\mathbb{R}^{2}$.

The solution of equation (2) is

$$
\begin{aligned}
x_{n} & =\frac{c_{1} \lambda_{0}^{n-1}+c_{2} \lambda_{-}^{n-1}+c_{3} \lambda_{+}^{n-1}}{c_{1} \lambda_{0}^{n}+c_{2} \lambda_{-}^{n}+c_{3} \lambda_{+}^{n}} \\
& =\frac{1}{\lambda_{-}} \frac{c_{1}\left(\frac{\lambda_{0}}{\lambda_{-}}\right)^{n-1}+c_{2}+c_{3}\left(\frac{\lambda_{+}}{\lambda_{-}}\right)^{n-1}}{c_{1}\left(\frac{\lambda_{0}}{\lambda_{-}}\right)^{n}+c_{2}+c_{3}\left(\frac{\lambda_{+}}{\lambda_{-}}\right)^{n}} .
\end{aligned}
$$

As $n \rightarrow \infty$,

$$
x_{n} \rightarrow \frac{1}{\lambda_{-}}=\bar{r}_{2} .
$$

\section{REFERENCES}

[1] R. Abo-Zeid, On the solutions of a second order difference equation, Math. Moravica, 21 (2) (2017), 61-73.

[2] R. Abo-Zeid, Global behavior of a higher order rational difference equation, Filomat, 30 (12) (2016), 3265-3276.

[3] R. Abo-Zeid, Global behavior of a third order rational difference equation, Math. Bohemica, 139 (1) (2014), 25-37.

[4] R. Abo-Zeid, Global behavior of a rational difference equation with quadratic term, Math. Moravica, 18 (1) (2014), 81-88. 
[5] R. Abo-Zeid, On the solutions of two third order recursive sequences, Armenian J. Math., 6 (2) (2014), 64-66.

[6] R. Abo-Zeid, Global behavior of a fourth order difference equation, Acta Commentaiones Univ. Tartuensis Math., 18 (2) (2014), 211-220.

[7] R. Abo-Zeid, Global behavior of a fourth order difference equation with quadratic term, Bol. Soc. Mat. Mex. (2017), https://doi.org/10.1007/s40590-017-0180-8.

[8] A. M. Amleh, E. Camouzis, G. Ladas On the dynamics of a rational difference equation, Part 2, Inter. J. Difference Equ., 3 (2) (2008), 195-225.

[9] A.M. Amleh, E. Camouzis, G. Ladas On the dynamics of a rational difference equation, Part 1, Inter. J. Difference Equ., 3 (1) (2008), 1-35.

[10] A. Anisimova, I. Bula, Some problems of second-order rational difference equations with quadratic terms, Inter. J. Difference Equ., 9 (1) (2014), 11-21.

[11] I. Bajo, Forbidden sets of planar rational systems of difference equations with common denominator, Appl. Anal. Discrete Math., 8 (2014), 16-32.

[12] I. Bajo, E. Liz, Global behaviour of a second-order nonlinear difference equation, J. Difference Equ. Appl., 17 (10) (2011), 1471-1486.

[13] I. Bajo, D. Franco, J. Perán, Dynamics of a rational system of difference equations in the plane, Adv. Difference Equ., 2011, Article ID: 958602, 17 pages.

[14] F. Balibrea, A. Cascales, On forbidden sets, J. Difference Equ. Appl., 21 (10) (2015), 974-996.

[15] V. I. Bogachev, Measure Theory, 1 \& 2, Springer Verlag, Berlin, 2006.

[16] E. Camouzis, G. Ladas, Dynamics of Third Order Rational Difference Equations: With Open Problems and Conjectures, Chapman \& Hall/CRC, Boca Raton, 2008.

[17] M. Dehghan, C. M. Kent, R. Mazrooei-Sebdani, N. L. Ortiz, H. Sedaghat, Dynamics of rational difference equations containing quadratic terms, J. Difference Equ. Appl., 14 (2) (2008), 191-208.

[18] P. R. Halmos, Measure Theory, Springer-Verlag, Berlin, 1974.

[19] E. A. Jankowski, M. R. S. Kulenović, Attractivity and global stability for linearizable difference equations, Comput. Math. Appl., 57 (2009), 1592-1607.

[20] J. J. Benedetto, W. Czaja, Integration and Modern Analysis, Birkhäuser Basel, 2009.

[21] C. M. Kent, H. Sedaghat, Global attractivity in a quadratic linear rational difference equation with delay, J. Difference Equ. Appl., 15 (10) (2009), 913-925.

[22] V. L. Kocic, G. Ladas, Global Behavior of Nonlinear Difference Equations of Higher Order with Applications, Kluwer Academic, Dordrecht, 1993.

[23] V. L. Kocic, G. Ladas, Global attractivity in a second order nonlinear difference equations, J. Math. Anal. Appl., 180 (1993), 144-150.

[24] M. R. S. Kulenović, M. Mehuljić, Global behavior of some rational second order difference equations, Inter. J. Difference Equ., 7 (2) (2012), 153-162.

[25] M. R. S. Kulenović, G. Ladas, Dynamics of Second Order Rational Difference Equations: With Open Problems and Conjectures, Chapman and Hall/HRC Boca Raton, 2002 .

[26] D. A. Salamon, Measures and Integration, ETH Zürich, 2016. 
[27] H. Sedaghat, On third order rational equations with quadratic terms, J. Difference Equ. Appl., 14 (8) (2008), 889-897.

[28] H. Shojaei, S. Parvandeh, T. Mohammadi, Z. Mohammadi, N. Mohammadi, Stability and convergence of a higher order rational difference equation, Austral. J. Bas. Appl. Sci., 5 (11) (2011), 72-77.

[29] I. Szalkai, Avoiding forbidden sequences by finding suitable initial values, Inter. J. Difference Equ., 3 (2) (2008), 305-315.

[30] J. C. Taylor, An Introduction to Measure and Probability, Springer-Verlag, New Yourk Inc., 1998.

[31] W. F. Trench, Introduction to Real Analysis, Prentice Hall, Upper Saddle River, 2002.

\author{
RaAfat Abo-Zeid \\ Department of Basic Science \\ The Higher Institute For \\ EngINEERING \& TECHNOLOGY \\ Al-Obour, Cairo \\ EGYPT \\ E-mail address: abuzead73@yahoo.com
}

